\title{
Curetagem cirúrgica associada à crioterapia como alternativa para tratamento de mixoma odontogênico em maxila: Relato de um caso clínico
}

\author{
Surgical curettage associated with cryotherapy as an alternative for the treatment of odontogenic
}

maxillary myxoma: Report of a clinical case
Legrado quirúrgico asociado a crioterapia como alternativa para el tratamiento del mixoma maxilar odontogénico: Reporte de un caso clínico

Recebido: 25/07/2021 | Revisado: 30/07/2021 | Aceito: 01/08/2021 | Publicado: 10/08/2021
Alana Oswaldina Gavioli Meira dos Santos ${ }^{1}$
ORCID: https://orcid.org/0000-0002-5591-5262
Universidade Federal de Mato Grosso do Sul, Brasil E-mail: nagavioli@gmail.com
Jose Carlos Garcia de Mendonça ${ }^{2}$ ORCID: https://orcid.org/0000-0003-3217-872X Universidade Federal de Mato Grosso do Sul, Brasil E-mail: jose.mendonca@ufms.br Gustavo Silva Pelissaro ${ }^{3}$
ORCID: https://orcid.org/0000-0003-3475-6001 Universidade Federal de Mato Grosso do Sul, Brasil E-mail: gustavopelissaro@ @otmail.com
Janayna Gomes Paiva Oliveira ${ }^{4}$
ORCID: https://orcid.org/0000-0003-2090-2872 Universidade Federal de Mato Grosso do Sul, Brasil
E-mail: janaynajgpaivaoliveira@gmail.com
Julio Cesar Leite da Silva ${ }^{5}$
ORCID: https://orcid.org/0000-0002-9835-7432 Universidade Federal de Mato Grosso do Sul, Brasil E-mail: julio.silva@ufms.com
Maisa de Oliveira Aguillera ${ }^{6}$
ORCID: https://orcid.org/0000-0003-0055-6984 Universidade Federal de Mato Grosso do Sul, Brasil E-mail: maisa.aguillera@live.com
Ellen Cristina Gaetti Jardim 7
ORCID: https://orcid.org/0000-0003-2471-465X
Universidade Federal de Mato Grosso do Sul, Brasil E-mail: ellen.jardim@ufms.com

\begin{abstract}
Resumo
O Mixoma Odontogênico é um tumor benigno raro, representando 3 a 5\% dos tumores odontogênicos de origem ectomesenquimal. Possui alta taxa de recidiva, principalmente por se tratar de um tumor não encapsulado. Acomete geralmente adulto jovem, em torno de 30 anos, com discreta predileção pelo sexo feminino. Assintomático em sua fase inicial, mas em lesões maiores pode causar descolamento ou mobilidade dental, disestesias e ulcerações. Radiograficamente apresenta-se como lesão radiolúcida, limites indefinidos, podendo haver trabéculas ósseas em seu interior (aspecto "bolha de sabão"). Não existe um protocolo para tratamento do tumor, uma vez que o mesmo pode-
\end{abstract}

\footnotetext{
${ }^{1}$ Mestranda do Programa de Pós-graduação em Ciências da Saúde da Universidade Federal de Mato Grosso do Sul.

${ }^{2}$ Coordenador da Residência em Cirurgia e Traumatologia Bucomaxilofacial da Faculdade de Odontologia da Universidade Federal de Mato Grosso do Sul.

${ }^{3}$ Doutorando do Programa de Pós-graduação em Ciências da Saúde da Universidade Federal de Mato Grosso do Sul.

${ }^{4}$ Preceptora da Residência em Cirurgia e Traumatologia Bucomaxilofacial da Faculdade de Odontologia da Universidade Federal de Mato Grosso do Sul.

5 Tutor da Residência em Cirurgia e Traumatologia Bucomaxilofacial da Faculdade de Odontologia da Universidade Federal de Mato Grosso do Sul.

${ }^{6}$ Mestranda do Programa de Pós-graduação em Ciências da Saúde da Universidade Federal de Mato Grosso do Sul.

${ }^{7}$ Tutora da Residência em Cirurgia e Traumatologia Bucomaxilofacial da Faculdade de Odontologia da Universidade Federal de Mato Grosso do Sul.
} 
se apresentar em mandíbula e maxila. Algumas literaturas relatam a eficácia do tratamento mais conservador como curetagem cirúrgica associada à crioterapia, para manutenção da função e estética do paciente. O presente trabalho visa apresentar o caso clínico de uma paciente do gênero feminino, feoderma, 38 anos de idade que apresentou Mixoma Odontogênico em maxila diagnosticado em 1997, sendo realizado o tratamento de curetagem cirúrgica associada à crioterapia, com acompanhamento de 23 anos onde a paciente não apresenta defeito ósseo significativo ou ausências dentárias, proporcionando a manutenção da função e estética facial.

Palavras-chave: Mixoma; Crioterapia; Tratamento Conservador.

\begin{abstract}
Odontogenic myxoma is a benign tumor, representing 3 a 5\% of odontogenic tumors of ectomesenchymal origin. It has high rates on recurrence, mainly for treating a nonencapsulated tumor. Usually accompanies Young adult, around 30 years old, with a slight predilection for the female sex. Asymptomatic in its initial phase, but larger lesions can cause dental detachment or mobility, dysesthesias and ulcerations. It presents radiografhically as a radiological lesion, indefinite limits, and there may be boné beamns inside ("soap bubble" aspect). There is no protocol for the treatment of tumors, since it can be presented in the mandible and maxilla. Some literature relates to more conservative treatment such as curgical curettage associated cryoterapy, to maintain the patient's function and aesthetics. The present work aims the clinical case of patient female, 38 years, who exhibits the Odontogenic Myxoma at the maximum diagnosis in 1997, with the treatment of surgical curettage associated cryoterapy being carried out, with monitoring of 23 years in which a patient does not present significant bone defector dental absences, wear and tear of the maintenance of function and facial aesthetics.
\end{abstract}

Keywords: Myxoma; Cryotherapy; Conservative Treatment.

\title{
Resumen
}

El mixoma odontogénico es un tumor benigno poco frecuente, que representa del 3 al 5\% de los tumores odontogénicos de origen ectomesenquimatoso. Tiene una alta tasa de recurrencia, principalmente porque es un tumor no encapsulado. Suele afectar a adultos jóvenes, en torno a los 30 años, con una ligera predilección por el sexo femenino. Asintomático en su fase inicial, pero en lesiones de mayor tamaño puede provocar desprendimiento o movilidad dentaria, disestesias y ulceraciones. Radiográficamente se presenta como una lesión radiotransparente, con límites indefinidos, pudiendo presentar trabéculas óseas en su interior (apariencia de "pompas de jabón”). No existe un protocolo para el tratamiento del tumor, ya que puede estar presente en mandíbula y maxilar. Alguna literatura reporta la efectividad de tratamientos más conservadores, como el legrado quirúrgico asociado a la crioterapia, para mantener la función y estética del paciente. El presente trabajo tiene como objetivo presentar el caso clínico de una paciente femenina, feoderma, de 38 años que presentó Mixoma odontogénico en el maxilar diagnosticado en 1997, realizándose el tratamiento de legrado quirúrgico asociado a crioterapia, con un seguimiento de 23 años. donde el paciente no presenta defecto óseo significativo o falta de dientes, proporcionando mantenimiento de la función y estética facial.

Palabras clave: Mixoma; Crioterapia; Tratamiento conservador.

\section{Introdução}

O Mixoma Odontogênico é um tumor benigno de origem odontogênica, porém, com característica de crescimento localmente agressivo (Moreira et al., 2011).

Trata-se de um tumor raro representando de 3 a 5\% dos tumores odontogênicos, de origem do ectomesenquima, não encapsulado levando a alta taxa de recidiva após o tratamento. Acomete em geral o osso mandibular de adultos jovens, 30 anos de idade e sem predileção por sexo (Neville et al., 2009).

As principais características clínicas do tumor são: crescimento assintomático, podendo causar deslocamento e mobilidade dentária em sua fase inicial. Em casos de lesões extensas pode gerar parestesia, ulcerações, expansão de cortical óssea e até acometimento de tecido mole adjacente (Brites, 2012).

As lesões pequenas em geral são diagnosticadas por achados radiográficos, apresentando uma lesão radiolúcida, bordas indefinidas, podendo conter trabéculas ósseas em seu interior (aspecto "bolha de sabão"). Tomograficamente observase lesão hipodensa, não delimitada, podendo causar expansão de corticais e trabéculas ósseas em seu interior. Por se tratar de um tecido mixóide, apresenta-se no exame de ressonância magnética como lesão de hiposinal em T1, não delimitada (Kawaekoga et al., 2014; Altug et al., 2011).

Os tumores de origem odontogênica possuem alta complexidade no que diz respeito ao tipo de tratamento adequado. 
O tratamento do Mixoma Odontogênico varia desde o emprego de técnicas conservadoras, como curetagem cirúrgica, até ressecção em bloco com margem de segurança (Rivera et al., 2019).

Não existe um protocolo estabelecendo o tratamento ideal, porém alguns autores referem a ressecção com margem de segurança como o ideal, levando em consideração a alta taxa de recidiva do tumor (Drumond, 2009). Em casos de tumores em sua fase inicial, a curetagem cirúrgica associada a tratamentos adjuvantes, como utilização de soluções químicas (Solução de Carnoy) ou crioterapia, mostra efetividade no tratamento e menor comorbidade pós-operatória ao paciente (Reverand et al., 2018).

A crioterapia vem sendo utilizada no tratamento de tumores benignos e malignos, quando utilizadas as temperaturas de $-20{ }^{\circ} \mathrm{C}$ a $-40{ }^{\circ} \mathrm{C}$, temperatura considerada ideal para causar a morte da célula residual no tecido. Podendo alcançar uma margem de, em média, $1 \mathrm{~cm}$ de penetração tecidual (Costa Jr et al., 2019).

A estética facial é um fator tão importante quanto a função do sistema estomatognático durante a decisão do tipo de tratamento a ser realizado em um paciente com Mixoma Odontogênico. Visto que, segundo Feitosa et al., 2009, o sorriso e o posicionamento dentário são um dos principais fatores levados em consideração para determinar a estética facial e autoestima do paciente.

Esse estudo tem por objetivo apresentar o caso de uma paciente portadora de Mixoma Odontogênico em Maxila, realizando-se um tratamento menos invasivo e uma abordagem mais conservadora sendo realizado curetagem cirúrgica associada a crioterapia.

\section{Metodologia}

Este trabalho é um estudo observacional com um braço único, com fins descritivos, exploratórios e com abordagem qualitativa, usando como base referencial Pereira (2018). Foram respeitados todos os procedimentos éticos correspondentes ao relato de caso, recebendo autorização e consentimento por escrito do paciente para as fotos pré, trans e pós-operatórias e o tratamento, assim como sua exposição científica.

\section{Caso Clínico}

Paciente do sexo feminino, 38 anos, feoderma, atendida no ambulatório de Cirurgia e Traumatologia Bucomaxilofacial do Hospital Universitário Maria Aparecida Pedrossian (HUMAP) da Universidade Federal de Mato Grosso do Sul (UFMS) em fevereiro de 2018, com queixa principal de incômodo em região de maxila e secreção de pus.

Durante anamnese paciente negou comorbidades sistêmicas, assim como alergia medicamentosa, tabagismo e etilismo. Referiu histórico de Mixoma Odontogênico (MO) em maxila esquerda diagnosticado no ano de 1997, e que após o diagnóstico foi submetida a 04 procedimentos cirúrgicos para curetagem da lesão.

O exame clínico extra-oral apresentou-se dentro da normalidade, com simetria facial e ausência de alterações sensoriais e/ou motoras (Figura 1). 
Figura 1: Aspecto facial da paciente. Ausência de assimetria.
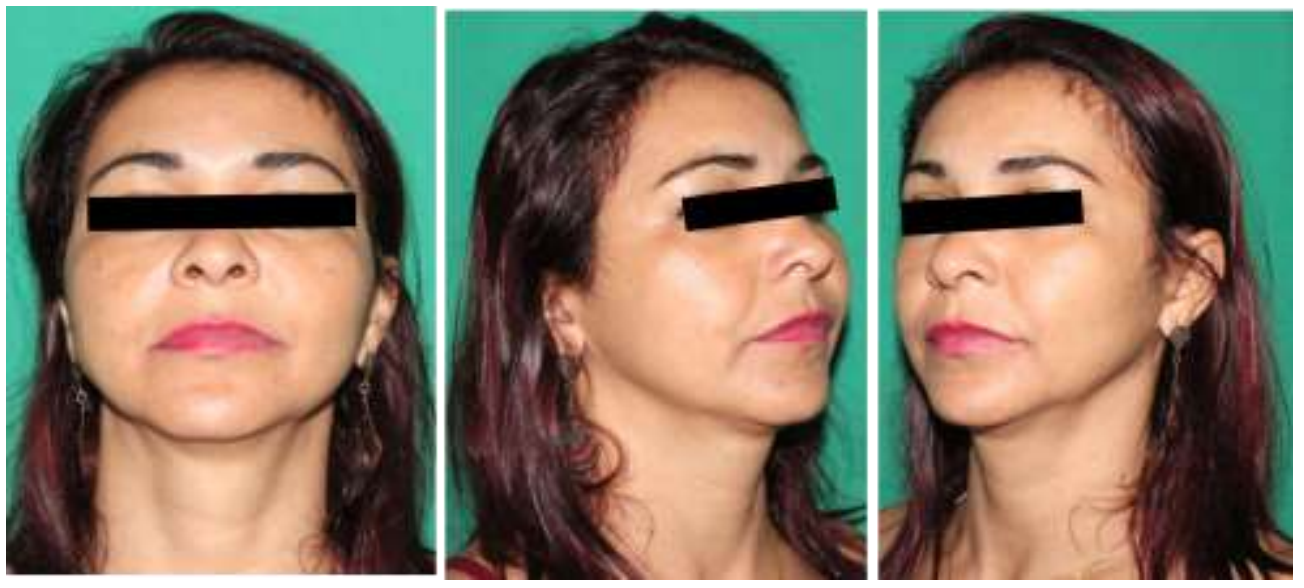

Fonte: Autores.

Ao exame clínico intra-oral observou-se oclusão dentária preservada com giroversão dos dentes 21 e 22, ausência dos dentes 45 e 46 e recessão gengival nos dentes 21 e 23. Drenagem de exudato purulento via sulco gengival do dente 23. Língua e mucosa de região mandibular e palato dentro da normalidade. Ausência de abaulamentro de cortical óssea vestibular, apenas discreta depressão em região de pilar canino esquerdo, presença de mucosa cicatricial em fundo de vestíbulo maxilar esquerdo, demais regiões de mucosa normocoradas e normotermas (Figura 2). À palpação paciente negou sensação dolorosa, apresentou mobilidade grau I nos dentes 21, 22, 23 e 24, os mesmos apresentando coloração escurecida, onde os dentes 21 , 22 e 23 com tratamento endodôntico já realizados e testes de vitalidade pulpar negativo para o dente 24.

Figura 2: Aspecto clínico intrabucal inicial.

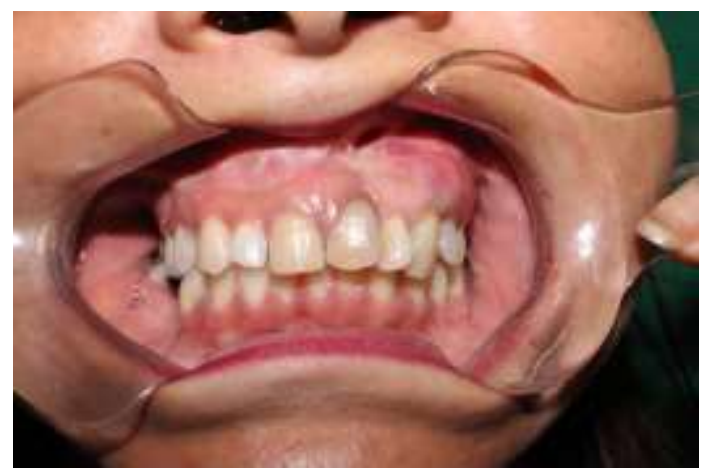

Fonte: Autores.

Em análise do exame tomográfico de março de 2018 notou-se lesão hipodensa com aspecto bolhoso acometendo maxila. No sentido latero-lateral com extensão de abertura piriforme do lado esquerdo até distal do dente 27 e em sentido antero-posterior desde maxila até palato duro, com envolvimento de parede lateral de cavidade nasal (Figura 3). 
Figura 3: Reconstruções axial, coronal, sagital e 3-D. Notar lesão hipodensa de aspecto bolhoso em região de maxila esquerda acometendo desde a abertura pirifome até a região de molar ipsilateral, com fenestração da tábua óssea vestibular.

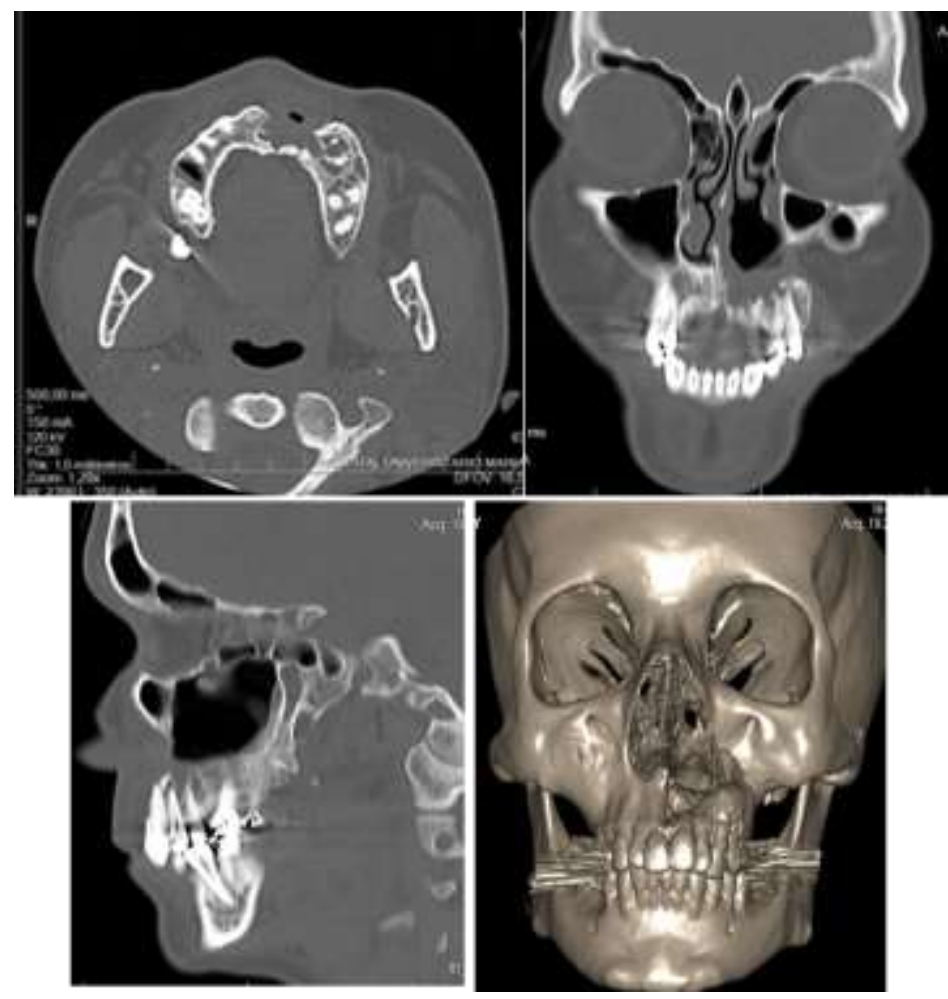

Fonte: Autores.

Os dados coletados do prontuário da paciente no HUMAP mostraram que em 1997 foi diagnosticado Mixoma odontogênico em maxila esquerda, confirmado por laudo anatomohistopatológico após realização de biopsia incisional.

De acordo com as evoluções contidas no prontuário, ao exame clínico extra oral a paciente apresentou assimetria facial causada por aumento volumétrico em região de maxila esquerda ocasionando apagamento do sulco nasolabial e ausência de selamento labial, assintomático e de evolução lenta. Intraoral apresentou aumento volumétrico em fundo de vestíbulo maxilar esquerdo, endurecido à palpação e assintomático (Figura 4). 
Figura 4: Imagens realizadas em 1997. Aumento volumétrico em hemiface esquerda e em fundo de vestíbulo ipsilateral.

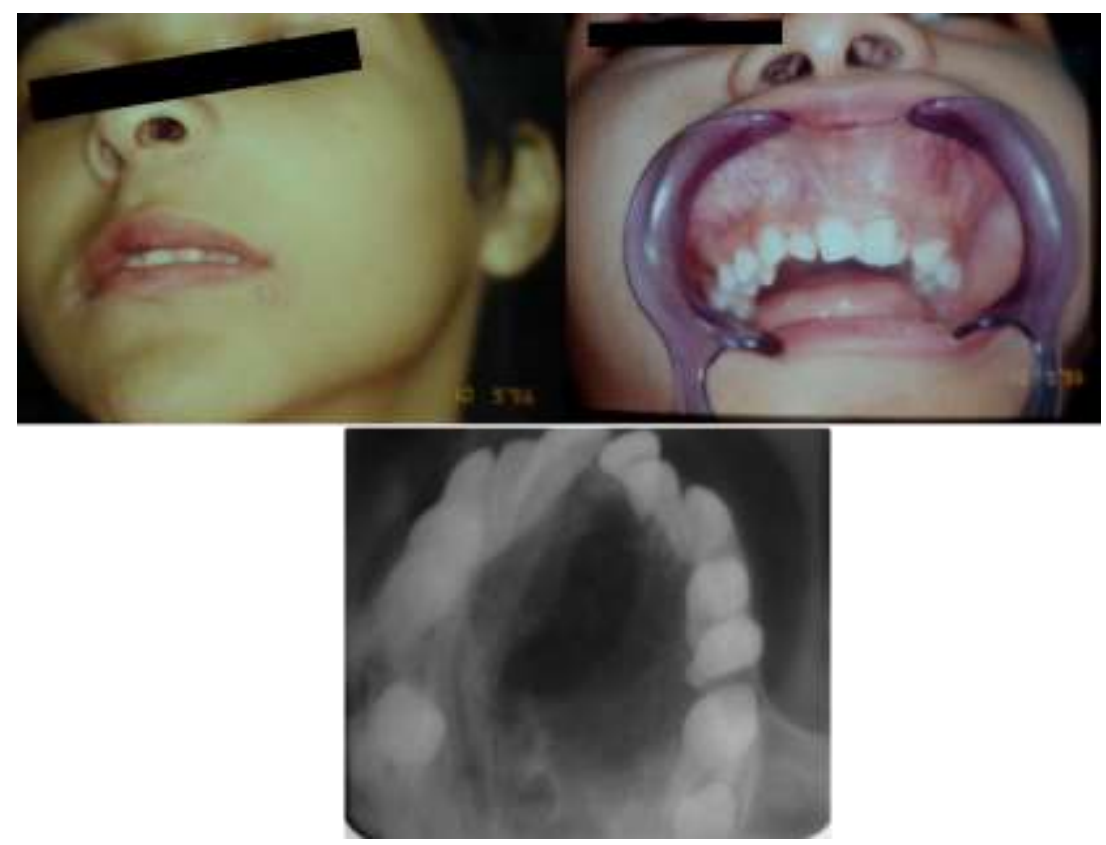

Fonte: Autores.

Ao exame radiográfico foi observada extensa lesão radiolúcida, com limites indefinidos, apresentando trabéculas ósseas em seu interior (aspecto "bolha de sabão") acometendo região anterior de maxila esquerda, com extensão de linha média desde abertura piriforme até mesial do dente 26.

Diante dos dados coletados, após análise das opções de tratamento, devido a idade da paciente no momento do diagnóstico, em 1997, considerando que a mesma estava em fase de crescimento e uma abordagem cirúrgica para a ressecção do tumor iria acarretar assimetria facial, alterações importantes na estética e função (fonação e deglutição, por exemplo) optouse pela realização de enucleação através da curetagem cirúrgica visando a manutenção das estruturas ósseas envolvidas pela lesão como tratamento, permitindo assim o completo desenvolvimento dos ossos faciais e estruturas adjacentes.

Em 2011 a paciente apresentou recidiva da lesão e foi realizada nova abordagem cirúrgica para curetagem, agora associada à crioterapia com utilização de Spray de gás refrigerante $\left(-50^{\circ} \mathrm{C}\right)$. Nos anos de 2012 e 2014 houve nova recidiva da lesão e realizou-se nova curetagem cirúrgica associada a crioterapia com Spray de gás refrigerante a $-50{ }^{\circ} \mathrm{C}$.

Após análise do histórico da paciente associado aos dados clínicos e tomográficos, a hipótese diagnóstica na consulta de retorno, em 2018, foi de recidiva de MO em maxila esquerda e infecção em seio maxilar esquerdo.

Diante do quadro apresentado o tratamento escolhido foi a antibioticoterapia para tratamento de infecção em seio maxilar esquerdo. Sendo realizado com Amoxicilina 500mg de 8 em 8 horas durante 07 dias e Metronidazol 250mg de 8 em 8 horas durante 07 dias. A paciente foi reavaliada após 07 dias, observando-se remissão total do quadro infeccioso.

Em abril de 2018 realizou-se curetagem cirúrgica do tumor em maxila esquerda associada a crioterapia com Spray de gás refrigerante a $-50{ }^{\circ} \mathrm{C}$ (Endo-Ice $\left.{ }^{\circledR}\right)$. O procedimento foi realizado sob anestesia geral, intubação nasoendotraqueal. Realizou-se a instalação de barra de Erich em maxila com objetivo de estabilizar os dentes da maxila. Após, foi feito o acesso cirúrgico do tipo Cadwell Luc em maxila esquerda seguido de curetagem da lesão em toda sua extensão (Figura 23 e 24) e posteriormente a realização da crioterapia, com aplicação do Spray de gás refrigerante a $-50{ }^{\circ} \mathrm{C}$ (Endo- Ice®) no leito cirúrgico durante 10 segundos, repetindo 3 vezes esse processo, com intervalo de 5 minutos entre cada aplicação(Figura 25). Realizouse a sutura da ferida operatória com fio Monocryl 4-0 (Figura 5). 
Figura 5: Aspecto trans-operatório após instalação da Barra de Erich em maxila. Notar acesso cirúrgico Caldwell Luc, fenestração óssea vestibular em maxila esquerda e presença de tecido tumoral intra-lesional. Crioterapia no leito cirúrgico, através da aplicação de Spray de gás refrigerante a $-50{ }^{\circ} \mathrm{C}$ (Endo-Iceß).
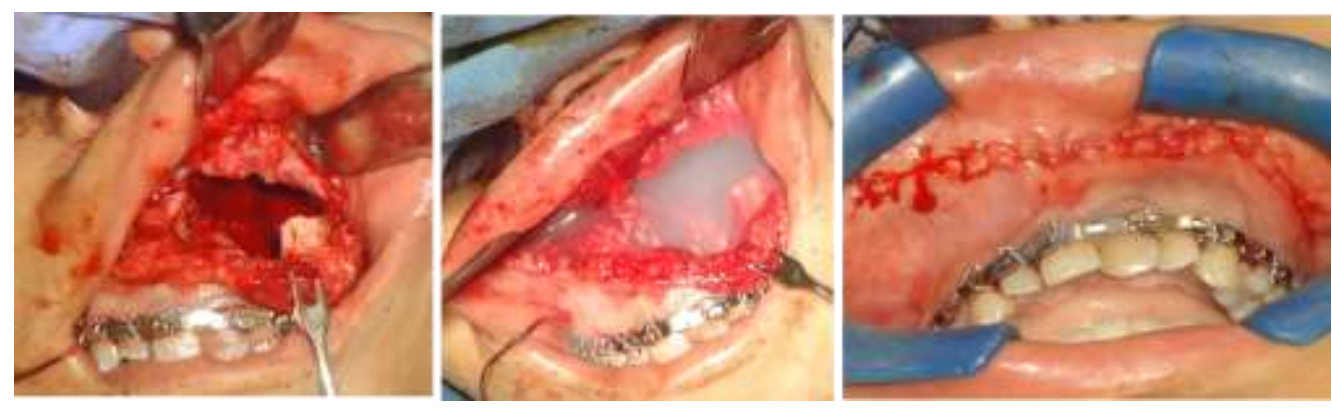

Fonte: Autores.

No pós-operatório imediato foram realizadas as seguintes medicações: Dexametasona por via intra venosa (IV) 10mg de 8 em 8 horas, Cefalotina IV 1000mg de 6 em 6 horas; Ranitidina IV 50mg de 12 em 12 horas, Ondasetrona IV 8mg de 12 em 12 horas e Dipirona IV 1000mg de 6 em 6 horas.

O laudo do exame anatomohistopatológico da lesão curetada na cirurgia confirmou diagnóstico de lesão mixóide compatível com MO.

Em avaliação pós-cirúrgica de 24 horas a paciente apresentou-se sem queixas álgicas, negou desconforto respiratório, sangramento, alteração de oclusão ou alterações sistêmicas. Ao exame clínico extra-oral observou-se edema em face do lado esquerdo, compatível com procedimento cirúrgico realizado, indolor à palpação. Intra-oral apresentou oclusão dentária preservada, sutura de acesso cirúrgico em posição adequada, ausência de hemorragia ou sinais flogísticos, higiene oral satisfatória. A paciente seguiu de alta hospitalar no segundo dia pós-operatório para acompanhamento ambulatorial.

Em retorno de 15 dias foi observado boa recuperação, com exame clínico extra-oral dentro da normalidade com remissão total de edema em face. Intra-oral observou-se boa cicatrização, higiene oral satisfatória, ausência de mobilidade dentária e ausência de sinais flogísticos. Foi realizada remoção de barra de Erich em maxila e sutura e a paciente foi encaminhada para realização de tratamento endodôntico dos dentes 23, 24, 25, 26 e 27 envolvidos na lesão.

Em acompanhamento de um ano e oito meses paciente relatou estar em tratamento odontológico para finalização da endodontia dos dentes maxilares esquerdos, conforme encaminhamento. Não houve alteração do exame clínico extra-oral (Figuras 31, 32 e 33). No aspecto clínico intra-oral notou-se recessão gengival do dente 21 e 23, ausência de mobilidade dentária, dentes 21, 22 e 23 com coloração escurecida, cicatrização de acesso cirúrgico apresentando áreas de fibrose, porém, sem fístula buco sinusal, ausência de sinais flogísticos e manutenção da oclusão (Figura 6). 
Figura 6: Paciente com 15 dias e 20 meses de pós-operatório, respectivamente. Observa-se simetria facial e ausência de anormalidades.
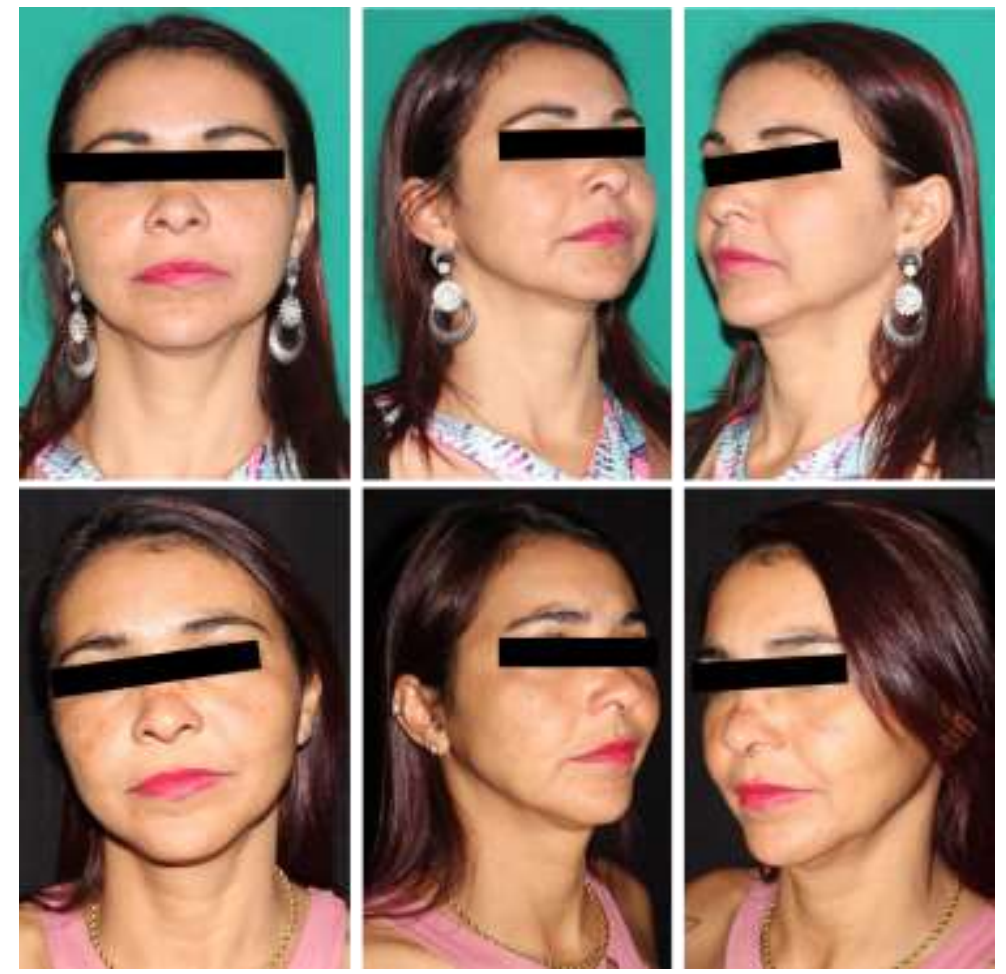

Fonte: Autores.

Ao exame tomográfico observou-se área de ausência óssea em maxila esquerda compatível com curetagem cirúrgica realizada, dentes 21, 22, 23, 24, 25 e 26 com tratamento endodôntico, osso remanescente com hiperdensidade dentro da normalidade, sem sinais de recidiva da lesão (Figura 7).

Figura 7: Reconstruções axial, coronal, sagital e 3-D de tomografia de face de 20 meses de pós-operatório. Ausência de recidiva de lesão em região de maxila esquerda, apresentando apenas fenestração de tábua óssea vestibular e defeito ósseo proveniente do último procedimento cirúrgico realizado.

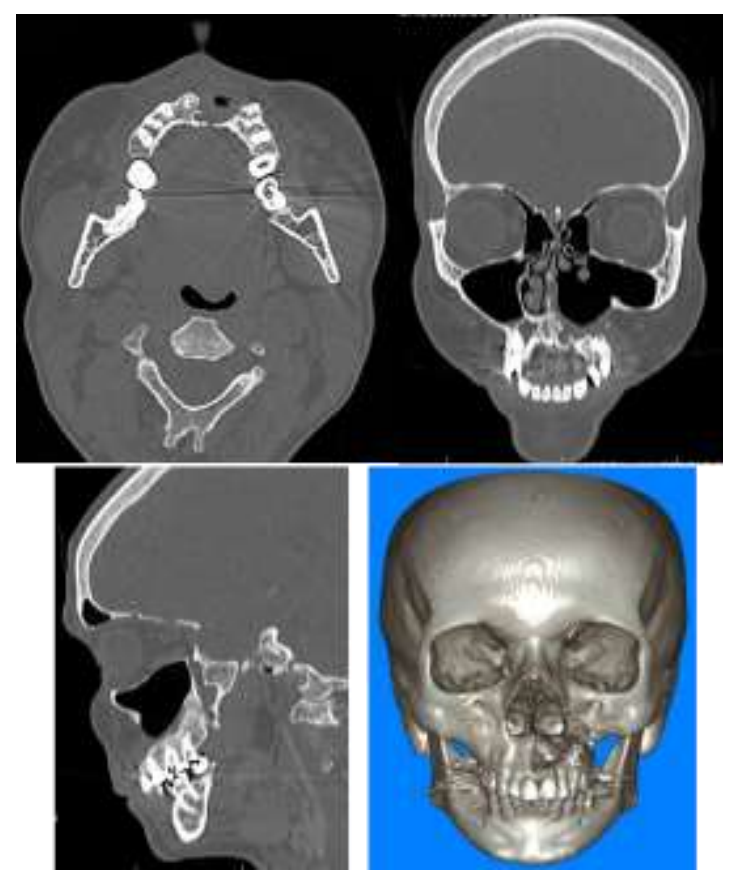

Fonte: Autores. 
A paciente segue em acompanhamento ambulatorial clínico e imaginológico, sem alterações clínicas e sem sinais de recidiva da lesão.

\section{Discussão}

A dificuldade do diagnóstico vem ao encontro com tipo de tratamento adequado para o Mixoma Odontogênico. Embora seja um tumor benigno, a sua característica de destruição local agressiva leva a uma abordagem mais ampla para seu tratamento.

O caso relatado foi de uma paciente do sexo feminino, adulta, apresentando lesão assintomática, assim como observados em alguns estudos (Zainine et al., 2014; Nonaka et al., 2007), embora alguns autores refiram que o MO não possui predileção por sexo (Cidel et al., 2011; Brites, 2012).

Como relatado por Radzinski, 2015, o MO é considerado o terceiro tumor de origem odontogênica mais recorrente (39\%), possui predileção pela região posterior da mandíbula, com crescimento lento e assintomático e alta taxa de recidiva por se tratar de um tumor não encapsulado, tem a capacidade de penetrar as trabéculas ósseas, causar destruição das corticais e perfurar e invadir tecidos moles adjacentes. O que enquadra o caso relatado nas características clínicas do tumor, no que diz respeito à recidiva e destruição óssea local, porém, ao contrário da literatura descrita, a localização do tumor era em maxila, o que nos mostra que não existe obrigatoriedade de região para o desenvolvimento dele.

O exame de TC da paciente mostrou uma lesão hipodensa em TC, gerando movimentação dentária (dentes 21 e 22 girovertidos) e fenestração de tábua óssea vestibular em maxila esquerda. Condizendo com Altug et al., 2011 que relataram em seu estudo as características imaginológicas do MO, como sendo uma lesão, hipodensa em TC, não delimitada, podendo causar deslocamentos, mas não gerando reabsorções dentárias, algumas vezes com septos ósseos em seu interior, o que apresenta um aspecto bolhoso "bolhas de sabão" e também podendo causar fenestração de tábua óssea.

Os tumores de origem odontogênica possuem características clínicas semelhantes, em alguns casos, o MO odontogênico, por exemplo, possui diagnóstico diferencial de ameloblastoma, fibroma odontogênico, granuloma central de células gigantes ou hemangioma central, entre outros (Ram et al., 2014). Como no caso relatado, as características clínicas e radiográficas sugeririam diagnóstico diferencial de Mixoma Odontogênico, Ameloblastoma ou Tumor Odontogênico Adenomatóide. Porém, devido ao diagnóstico inicial no ano de 1997 e os registros de recidiva, o diagnóstico clínico foi de Mixoma Odontogênico, confirmado no exame anatomohistopatológico.

Embora fosse um quadro de recidiva, comum para esse tipo de tumor (Nunes et al., 2021), onde autores como Rivera et al. 2019, indicam ressecção com margem de segurança, optou-se por nova curetagem cirúrgica associada à crioterapia, pois, como relatado por Costa et al., 2010, em casos de recidiva local sem complicações a reabordagem para realização de nova criocirurgia é viável, uma vez que a ressecção levaria a um defeito funcional e estético importante na maxila.

A utilização do Spray de gás refrigerante a $-50{ }^{\circ} \mathrm{C}$ foi eleita pela boa margem cirúrgica alcançada pelo congelamento do tecido, cerca de $2 \mathrm{~cm}$, a viabilidade financeira e empregabilidade da técnica, conforme o estudo de Costa Jr. et al., 2018 que mostrou a utilização da técnica do spray em cavidade cirúrgica com eficácia, precisão e segurança de utilização em tecido ósseo e tecido mole. De acordo com Moraes et al. 2015, a utilização da crioterapia gera uma margem de aproximadamente $1 \mathrm{~cm}$ a partir da área aplicada.

Optou-se pela realização do tratamento endodôntico dos dentes envolvidos na lesão, uma vez que o suprimento vásculo-nervoso dos mesmos havia sido removido no momento da curetagem cirúrgica, podendo assim, os canais dentinários serem uma via de contaminação para a região operada. 
Considerando um tratamento iniciado há 23 anos, tendo a paciente um desenvolvimento facial completo sem alterações em seu cotidiano e convivência social. Há 01 ano e 08 meses não apresenta sinais de recidiva, mantendo as atividades funcionais como mastigação, fonação e deglutição e também estéticas e convívio social.

A face é uma das áreas mais importantes do corpo humano, no que diz respeito a auto-estima e convivência social. Considera-se a viabilidade do tratamento realizado, sem prejuízos à paciente, pois a mesma manteve sua aparência e funções do sistema estomatognático sem alterações, e principalmente, sem danos psicossociais. Uma vez que na pesquisa apresentada por Feitosa et al. 2009, os dentes e o sorriso são considerados os aspectos mais importantes para a estética e autoestima do paciente.

\section{Conclusão}

O estudo mostrou que o tratamento do Mixoma Odontogênico é complexo e depende diretamente da extensão e localização da lesão e da experiência do cirurgião. Embora existam diversas formas de tratamento, a maioria dos autores opta pela ressecção do tumor, o que levaria a grandes defeitos ósseos.

O caso relatado demonstrou que é possível realizar um tratamento mais conservador de mixoma em maxila, desde que haja seguimento, evitando seqüelas, possibilitando assim a manutenção da função, estética e a qualidade de vida da paciente.

\section{Agradecimentos}

O presente trabalho foi realizado com apoio da Fundação Universidade Federal de Mato Grosso do Sul - UFMS/MEC - Brasil.

\section{Referências}

Alchalabi, N. J., Merza, A. M., \& Issa, S. A. (2017). Using Crnoy’s Solution in Tratamento of Keratocystic Odontogenic Tumor. Annals of Maxillofacial Surgery. 7(1), 51-56.

Altug, H. A., Gulses, A., \& Sencimen, M. (2011). Clinico-radiographic Examination of Odontogenic Myxoma with Displacement of Unerupted Upper Third Molar: Review of the Literature. International Journal. Morphology. 29(3), 930-933.

Brites, F. C. (2012). Mixoma odontogênico - tratamento cirúrgico radical. Revista de Cirurgia e Traumatologia Buco-Maxilo-Facial. 12(4), 33-38.

Cidel, L. E. E., Rodríguez, R. R., \& Taylor, A. M. (2011). Mixoma odontogénico. Presentación de caso clínico. Revista Odontológica Mexicana. 15(4), 244250 .

Chrcarnovic, B. R., Amaral, M. B. F., Marigo, H. A., \& Freire-Maia, B. (2010). Na expanded odontogenic myxoma in maxila. Stomatologija. 12(4), 122-128.

Costa, F. W. G., Soares, E. C. S., \& Batista, S. H. B. (2010). Criocirurgia no tratamento de lesões benignas dos maxilares: revisão de literatura e análise de 103 casos previamente reportados. Revista Sul-Brasileira de Odontologia. 7(2), 208-15.

Costa Jr, A. S., Miotto, A., Paulo, G. A., Ferrari, A. P., \& Ota, L. H. (2019). Experimental study for evaluation of a low-cost spray cryoterapy system. Eistein. $17(2), 1-5$.

Drumond, J. M. N. (2009). Tumores ósseos benignos e lesões ósseas pseudotumorais: tratamento atual e novas tendências. Revista Brasileira de Ortopedia. 44(5), 386-90.

Feitosa, D. A. S., Dantas, D. C. R. E., Guênes, G. M. T., et al. (2009). Percepção de pacientes e acadêmicos de odontologia sobre estética facial e dentária. Revista Fluminense de Odontologia. 14(1), 23-26.

Gomes, C. C., Diniz, M. G., Duarte, A. P., Bernardes, V. F., \& Gomez, R. S. (2011). Molecular review of odontogenic myxoma. Oral Oncology. 47, $325-328$.

Guerra, L. A. P., Silva, P. F., Santos, R. L. O., Silva, A. M. F., \& Albuquerque, D. P. (2013). Tratamento conservados de múltiplos tumores odontogênicos ceratocísticos em paciente não sindrômico. Revista de Cirurgia e Traumatologia Buco-Maxilo-Facial, Camaragibe. 13(2), 43-50.

Hupp, J. R., Ellis III, E., \& Tucker, M. R. (2009) Cirurgia Oral e Maxilofacial Contemporânea. Elsevier.

Kawase-Koga, Y., Saijo, H., Hoshi, K., Takato, T., \& Mori, Y. (2014). Surgical management of odontogenic myxoma: a case report and review of the literature. BMC Research Notes. 7, 214-20.

Llerandi, J. V., Pita, A. J., \& Gonzáles, M. C. F. (2009). Mixoma odontogénico. Revista Cubana de Estomatologia. 46(3), 62-69. 
Research, Society and Development, v. 10, n. 10, e103101018693, 2021

(CC BY 4.0) | ISSN 2525-3409 | DOI: http://dx.doi.org/10.33448/rsd-v10i10.18693

Moraes, P. C., Teixeira, R. G., Thomaz, L. A., Junqueira, J. L. C., \& Oliveira, L. B. (2015). Cryosurgery for the treatment of pediatric plunging ranula: a conservative manegement. Revista Gaúcha de Odontolgia. 63(4), 492-495.

Moreira, P. R., Cardoso, F. P., Brito, J. A. R., Batista, A. C., Gomes, C. C., \& Gomez, R. S. (2011). Hypomethylation of tumor suppressor genes in odontogenic myxoma. Brazilian Dental Journal. 22(5),422-427.

Neville, B. W., Damm, D. D., Allen, C. M., \& Bouquot, J. E. (2009) Patologia Oral e Maxilofacial. Elsevier.

Nonaka, C. F. W., Cavalcante, R.B., Piva, M. R., Souza, L. B., \& Pinto, L. P. (2007) Mixoma odontogênico: estudo clínico-patológico de 14 casos. Ciência Odontológica Brasileira. 10(2), 61-67.

Nunes, C. E. N., Morais, A. D. A., Lima, J. V. L., Chaves, F. N., Sampieri, M. B. S., \& Oliveira, D. H. I. P. (2021). Peripheral odontogenic myxoma: Clinical case with a literature review. Research, Society and Development. 10(6), 1-8.

Pereira, P. A. 2018. Metodologia da pesquisa científica [recurso eletrônico]. [et al.]. - 1. ed. - Santa Maria, RS: UFSM, NTE.

Pogrel, M. A., Podlesh, S., Anthony, J. P., \& Alexander, J. (1997). A Comparison of Vascularizedand Nonvascularized Bone Grafts for Reconstruction of Mandibular Continuity Defects. Journal of Oral and Maxillofacial Surgery. 55, 1200-1206

Radzinski, F. K. (2015). Mixoma mandibular. Revisión bibliográfica. A propósito de 2 casos clínicos. Odontoestomatologia. 17(26), 65-77.

Ram, H., Mehta, G., Manoj, K., \& Lone, P. (2014). Odontogenic myxoma in a 52-year-old woman. BMJ Case Reports. 2014:bcr2013202416.

Reverand, S. D, Gías, L. N., Guerra, M. M., García, R. G., Pérez, J. S., \& Campo, F. J. R. (2018). Mixoma odontogénico: presentación de uma serie de 4 casos clínicos y revisión de la literatura. Revisa Española de Cirurgía Oral Y Maxilofacial. 40(3), 120-128.

Rivera, G. J. G., Ortega-Pertuz, A. I., \& Suarez, M. L. E. (2019). Fibromixoma Odontogénico Agressivo em Maxila: Abordaje Quirúrgico. International Journal of Odontostomatology. 13(1), 58-63.

Sharif, F. N. J., Oliver, R., Sweet, C., \& Sharif, M. O. (2015). Interventions of the tratamento f keratocystic odontogenic tumours (Review). Cochrane database of systematic reviews. 11(cd008464), 1-11.

Speight, P. M., \& Takata, T. (2018). New tumour entities in the 4th edition of the World Health Organization Classification of Head and Neck tumours odontogenic and maxillofacial bone tumours. Virchows Archiv. 472, 331-339.

Vasconcelos, A. C. U., Silveira, F. M., Gomes, A. P.N., Tarquinio, S. B. C., Sobral, A. P. N., Arruda, J. A. A., et al. (2018). Odontogenic myxoma: A 63-year retrospective multicenter study of 85 cases in a Brasil population and a review of 999 cases from literature. Journal of Oral Pathology and Medicine. 47, 71-7.

Zainine, R., Mizouni, H., El Karbi, A., Beltaief, N., Sahtout, S., \& Besbes, G. (2014). Myxome de l'os maxillaire. Annales d'Oto-Laryngologie et de Chirurgie Cervico-Faciale. 131, 243-245. 